\title{
Boosted Top Quarks, Top Pair Resonances, and Top Partner Searches at the LHC
}

\author{
Justin Pilot ${ }^{1, a}$, on behalf of the ATLAS and CMS Collaborations \\ ${ }^{1}$ University of California, Davis
}

\begin{abstract}
The increased center-of-mass energy of proton-proton collisions at the LHC allows for higher and higher mass scales to be probed in the search for new physics. These searches for new physics can often involve the reconstruction of top quark decays. In this high-mass regime, event topologies can change and top quark decay products become highly boosted, requiring the use of special identification algorithms. This report summarizes the latest techniques used in the reconstruction of boosted top quarks, and highlights several new physics searches from ATLAS and CMS where they are utilized. Such analyses include the search for top quark pair resonances as is expected from $Z^{\prime}$ decays, as well as top quark partner searches, such as fourth generation vector-like quarks, excited top quarks, and top quark partners with non-standard charges.
\end{abstract}

\section{Introduction}

The Large Hadron Collider (LHC) can truly be thought of as a top quark factory. With the completion of the running period in 2012, we have now obtained an enormous data sample of top quarks. This sample allows the analysis of many independent decay channels and provides a robust understanding of top quark reconstruction at the LHC experiments. This provides a perfect final state to use in the search for new physics.

Many models of new physics have large couplings to third-generation particles, including the top quark. These models, including Randall-Sundrum Kaluza-Klein gluons [1], $Z^{\prime}$ particles [2], and exotic partners to the top quark, can provide a solution to the hierarchy problem without the introduction of supersymmetry. In addition, the unique event signatures provided by these models, which include $b$ quark jets, boosted top quarks or vector bosons in the final state, can enhance the sensitivity of searches greatly.

These topologies can be reconstructed through the use of specialized algorithms to reconstruct highly-boosted top quark decays, where particles become extremely collimated and would fail to be reconstructed properly using standard techniques. These new techniques can push further the mass reach of searches for new physics.

This report summarizes a plenary talk given at the 2013 LHCP conference held in Barcelona, Spain. The latest boosted top quark algorithms will be presented, along with the performance obtained using data obtained in both the 2011 and 2012 running of the LHC. After presenting details of the specific algorithms used by the ATLAS [3] and CMS [4] experiments, their use in physics analysis will be detailed. Several analyses will be covered, including searches for $t \bar{t}$ resonances in various decay modes,

\footnotetext{
ae-mail: jrpilot@ucdavis.edu
}

searches for vector-like $t^{\prime}$ quarks, exotic top quarks with electric charge $5 e / 3$, and finally excited top quarks $t^{\star}$. Many of the analyses detailed here were shown publicly for the first time at the LHCP conference in May 2013.

\section{Boosted Top Quarks}

When reconstructing top quarks in a search for new physics, different strategies apply based on the mass range of interest to the analysis. For an example particle decay $X \rightarrow t \bar{t}$, there are three regimes to consider. For low mass $\left(m_{X}<1 \mathrm{TeV} / c^{2}\right)$ searches, the decay products of the top quarks will be well-separated, and standard top quark reconstruction methods can be utilized. Leptons will be isolated, and there is a one-to-one correspondence between quarks from hadronic top or $W$ boson decays and reconstructed jets in the detector.

When entering the high-mass regime $\left(m_{X}>2 \mathrm{TeV} / c^{2}\right)$, the top quarks become boosted. Their decay products become collimated, with an angular separation $\Delta R$, which goes roughly as $\Delta R \sim 2 m_{t} / p_{T}$, where $p_{T}$ is the transverse momentum of the boosted top quark. This can influence the choice of the reconstructed jet distance parameter (cone size) used in the analysis, ensuring that all the decay products can be 'caught' by a single large-sized jet. With this choice, one can use specialized algorithms to identify highly-boosted top quarks which are reconstructed in a single jet. These algorithms, known as 'top-tagging' algorithms, use jet substructure information to identify the top quark decay topology.

Even with the large-sized jets used in boosted top quark reconstruction, in the intermediate mass range (1 $\mathrm{TeV} / c^{2}<m_{X}<2 \mathrm{TeV} / c^{2}$ ), a mix of techniques needs to be utilized. For example, if a top quark is not sufficiently boosted, only the decay products of the $W$ boson 


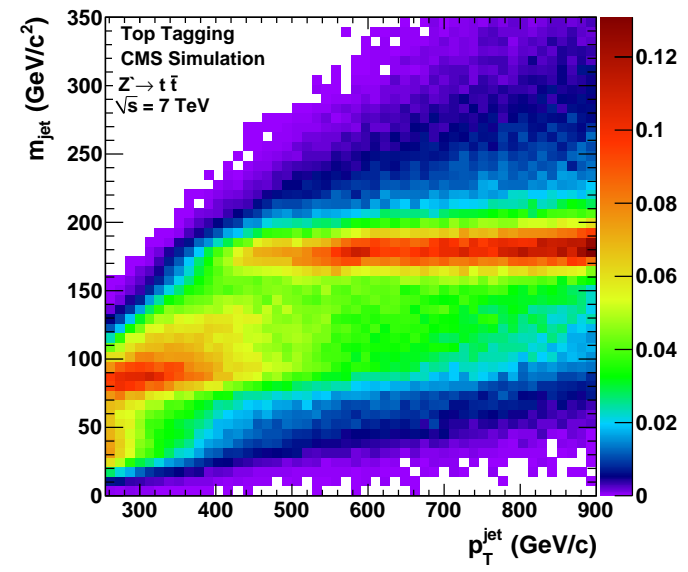

Figure 1. Two-dimensional plot of jet mass versus jet $p_{T}$, showing partially merged (low $p_{T}$ ) and fully merged (high $p_{T}$ ) top quarks which have collimated decay products reconstructed within single jets. This plot includes events with $Z^{\prime}$ masses ranging from 750 to $4000 \mathrm{GeV} / c^{2}$. Figure from Ref. [5].

may merge into a single jet, while the $b$-quark jet will be reconstructed separately. Again, there are specialized algorithms to reconstruct hadronic decays of vector bosons, which can be utilized in this case. Special techniques are also in order for leptonic decays. For example, the lepton from a boosted top quark decay may overlap with the $b$-quark jet from the same decay. In this case, standard lepton isolation requirements may veto the event, when this is obviously not what should be done.

Figure 1 illustrates the different regimes in simulated fully-hadronic $Z^{\prime} \rightarrow t \bar{t}$ decays - for lower $p_{T}$ jets, the jet mass distribution shows a peak at the $W$ mass. Here, the $W$ boson decay products are merged into a single jet, hence the peak at the $W$ mass for single-jet masses is obtained. With increasing jet $p_{T}$, a transition is observed and the peak shifts to the mass of the top quark. This occurs when all decay products are sufficiently collimated to be reconstructed in a single jet.

This section will summarize a few of the most common types of reconstruction algorithms which are used for boosted hadronic top decays, before detailing their use in specific analyses and searches for new physics.

\subsection{HEP Top Tagger}

The HEP Top Tagger [6] is an algorithm used extensively by the ATLAS Collaboration for physics analysis. The HEP Top Tagger uses a large jet distance parameter, typically $R=1.5$, as a starting point to reconstruct boosted top decays. As this is much larger than standard jets used in both the ATLAS and CMS experiments, the algorithm can be used for identifying even moderately boosted top quarks with $p_{T} \sim 200 \mathrm{GeV} / c$. The Cambridge-Aachen clustering algorithm is used to define these large jets used for the algorithm.

Constituents of the original, large $R=1.5$ jet are reclustered into a set of hard subjets, in the process removing constituents which are not due to hard-scale splittings,
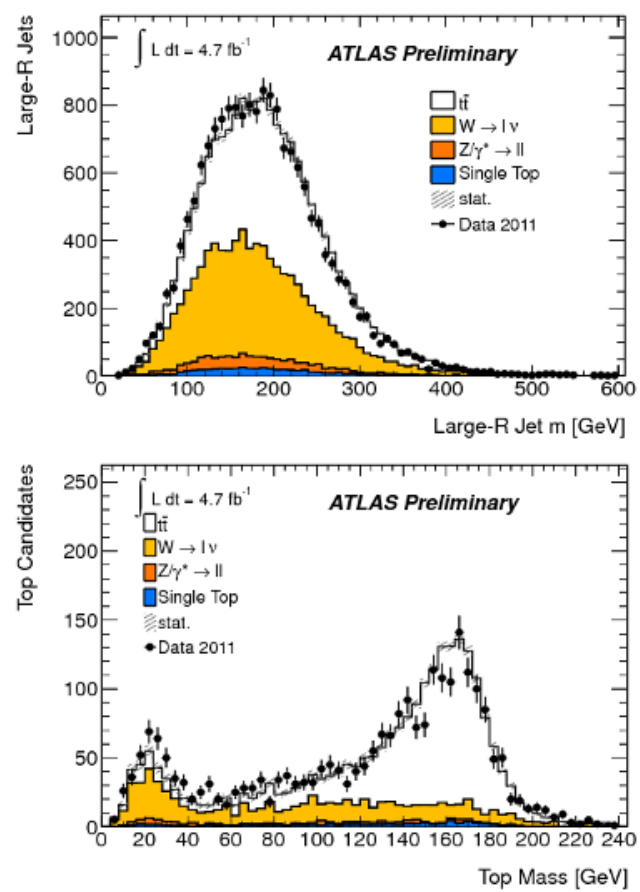

Figure 2. Single jet mass distribution before (top) and after (bottom) the HEP Top Tagger selections are applied. Afterwards, a peak is seen at the top quark mass, and a pure sample of boosted top quarks is obtained using this algorithm. Figures from Ref. [7].

such as from soft or wide-angle radiation. After the reclustering, only the five hardest subjets are retained. These five subjets are then further reclustered until only three objects remain. These three objects are then the three candidate decay products of a boosted top quark.

These three combinations are checked for consistency with a top quark. The overall mass of the three subjets must be consistent with the top quark mass, and there must be a combination of two subjets which gives the $W$ boson mass. There are other criteria based on the pairwise subjet combinations as well.

The HEP Top Tagger has an identification efficiency of roughly $40 \%$ for top quarks with $p_{T}>400 \mathrm{GeV} / c$, and can be used to identify merged top quark decays at $p_{T}$ values as low as $200 \mathrm{GeV} / c$ due to the large jet size used. The efficiency for background events is just a few percent. Figure 2 shows jet mass distributions before and after the HEP Top Tagger selections are applied. A clean sample of top quarks is obtained after the full selection.

\subsection{CMS Top Tagger}

Similarly to the HEP Top Tagger, the CMS Top Tagger aims to identify the three subjets corresponding to the decay products of the top quark. It is based on the top tagging algorithm developed at Johns Hopkins University [8]. The algorithm uses a smaller jet size, $R=0.8$, and obtains subjets by reversing through the original jet pairwise clustering sequence. Again, the Cambridge-Aachen clustering 


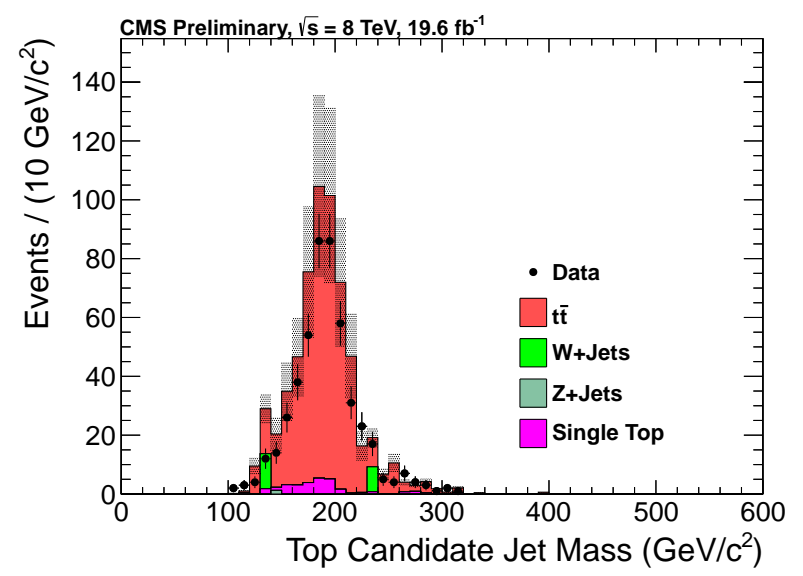

Figure 3. Jet mass distribution for fully-merged top quark candidates, identified with the CMS top tagging algorithm. Figure from Ref. [9].

algorithm is used to define the jets used here. A jet or subjet can be decomposed to its two parents in the clustering sequence, if the two are widely separated and the transverse momenta of each are sufficiently large. Again, the aim is to remove constituents due to soft and large-angle radiation within the jet. The process is repeated up to two times, resulting in a possible maximum of four identified subjets.

To identify a top quark, jets with three or four identified subjets are used. Again, the total mass of the jet is required to be consistent with the top quark mass. To identify the combination of subjets corresponding to the $W$ boson decay, the pair of subjets giving the minimum mass is used. A cut on this quantity, $m_{\min }>50 \mathrm{GeV} / c^{2}$, is used in the algorithm. The efficiency of the CMS top tagging algorithm is roughly $50 \%$ at high $p_{T}(>400 \mathrm{GeV} / c)$, with a mistag rate of less than $10 \%$. Because of the smaller jet size used, the efficiency drops off very quickly at lower values of $p_{T}$.

This tagging algorithm is used by the CMS experiment, and has been extensively validated in data. Figure 3 shows the top-tagged jet mass distribution in a sample of boosted top quarks obtained using this algorithm from the CMS experiment. Good agreement is observed between data and simulated events.

\section{Physics Analyses}

There are several analyses utilizing the special techniques described above, exploiting the unique topologies of top quark decays in the final state. This section describes some of the latest results in this area of study.

\subsection{Top Pair Resonances}

Both the ATLAS and CMS collaborations search for resonances using the reconstructed top quark pair invariant mass $\left(m_{t \bar{t}}\right)$ spectrum. These searches are sensitive to new particles decaying to top quark pairs, such as $Z^{\prime}$ bosons of various resonance widths, or RS KK gluons. The searches are based a mix of techniques, as is necessary for the range of masses being explored. In the high-mass regime, these searches rely heavily on jet substructure techniques. The top pair resonance searches include all possible decay channels: all-hadronic, lepton+jets, and dilepton topologies.

The CMS search for $t \bar{t}$ resonances in the lepton+jets topology [10] uses a standard top reconstruction for lowmass searches, requiring 4 or more jets with $p_{T}$ requirements of $70,50,30,30 \mathrm{GeV} / c$. One isolated high- $p_{T}$ electron or muon is identified, at least one $b$-jet is required, along with missing transverse energy greater than $20 \mathrm{GeV}$. For high-mass searches, events with only 2 jets $\left(p_{T}>150\right.$, $50 \mathrm{GeV} / c$ ) can enter the selection - one fully merged top quark and the $b$ quark jet from the leptonically decaying top quark. The isolation requirement is relaxed on the lepton identification in this case due to the boosted topology. Requirements on the missing transverse energy are increased, and an $H_{T}^{l e p}>150 \mathrm{GeV}$ requirement is imposed, where $H_{T}^{\text {lep }}$ is the scalar sum of the missing transverse energy and the lepton transverse momentum. This differs sligtly from $H_{T}$, the scalar sum of jet and lepton transverse momenta.

After identifying events consistent with the expected event signature, $\chi^{2}$ function is computed to determine assignment of jets to top quark candidates, using constraints on the top quark and $W$ boson masses and other kinematic properties of the signal process. After the minimization of this $\chi^{2}$ function, the $m_{t \bar{t}}$ distribution is formed and used for signal discrimination.

The ATLAS search in the lepton+jets channel [11] is similar in that it uses two different selections for the two mass regimes of interest. An isolated electron or muon is selected $\left(p_{T}>25 \mathrm{GeV} / c\right)$, along with missing transverse energy above 20 (25) $\mathrm{GeV}$ for the electron (muon) channel. For the low-mass regime, the 'resolved' selection requires 3 or more jets with $p_{T}>25 \mathrm{GeV} / c$ if one jet has a mass $m>60 \mathrm{GeV} / c^{2}$. If this is not the case, 4 jets are required. Similarly to the CMS search, this search also uses a $\chi^{2}$ algorithm for event reconstruction. In the boosted topology, a large $R=1.0$ jet is used, requiring it to have $p_{T}>350 \mathrm{GeV} / c, m>100 \mathrm{GeV} / c^{2}$. An additional requirement is imposed on the $k_{T}$ splitting scale, a measure of the energy of the final combination of the jet clustering process. The search also requires at least one jet to be consistent with a $b$ quark (it could overlap with the large jet used for boosted top quark identification. Again the $m_{t \bar{t}}$ distribution is used for signal discrimination, after combining the resolved and boosted sub-analyses.

Using $14.3 \mathrm{fb}^{-1}$ of integrated luminosity, ATLAS excludes $Z^{\prime}$ bosons (narrow width) with masses up to 1.8 $\mathrm{TeV} / c^{2}$, and $\mathrm{KK}$ gluons with masses up to $2.0 \mathrm{TeV} / c^{2}$. CMS, using $19.6 \mathrm{fb}^{-1}$, excludes narrow (1\% width) $Z^{\prime}$ up to $2.1 \mathrm{TeV} / c^{2}$, and $\mathrm{KK}$ gluons (with slight differences in signal models from ATLAS) up to $2.5 \mathrm{TeV} / c^{2}$. CMS also studies a wide (10\% width) $Z^{\prime}$ signal model, excluding masses in this scenario up to $2.7 \mathrm{TeV} / c^{2}$. Figure 4 shows a comparison of the limits obtained by ATLAS and CMS 

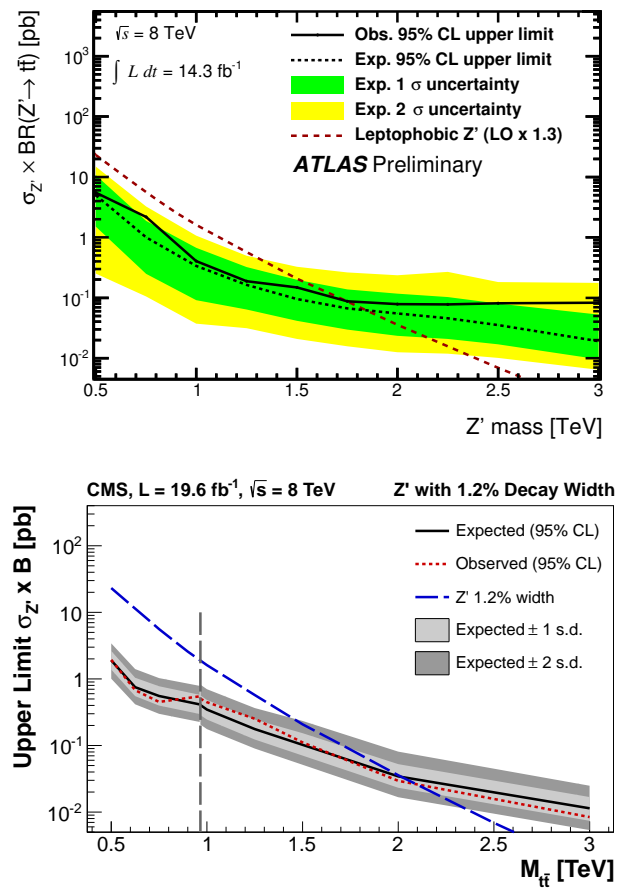

Figure 4. Comparison of limits on a narrow-width $Z^{\prime}$ boson obtained by ATLAS (top, figure from [11]) and CMS (bottom, figure from [10]).

in this channel, for the $Z^{\prime}$ signal hypothesis having a width of about $1 \%$ of its mass.

Searches in the dilepton decay mode are also performed by both the ATLAS and CMS collaborations, but as of this writing have not been updated with the $\sqrt{s}=8$ $\mathrm{TeV}$ collision data from 2012. The exclusion limits reach to roughly $1 \mathrm{TeV} / c^{2}$, depending on the signal model considered. For more information, please see the analysis documentation [12, 13].

Searches in the all-hadronic decay mode intensely use the top tagging algorithms described above. The ATLAS search in this channel [14] uses the HEP Top Tagger as well as an additional algorithm, the template tagger, which examines the distribution of energy deposits within a jet and determines their consistency with expectations (templates) from hadronic top quark decays. This search also requires a $b$-tagged jet to be reconstructed near the large jet used for top quark identification. Again, the $m_{t \bar{t}}$ distribution is used to set limits on various new physics processes.

The CMS all-hadronic search [9] uses the CMS Top Tagger described above, in combination with a requirement on the rapidity separation, $\Delta y$, of the two top quark candidate jets, which serves to further enhance the sensitivity of this analysis in the high-mass regime. The main background in this search is non-top multijet production, which is estimated using a data-based method. This differs from the ATLAS search, for which the main background is Standard Model $t \bar{t}$ production. This is due to the details of the different top tagging algorithms used. Figure 5 compares the $m_{t \bar{t}}$ distributions used in each analysis.
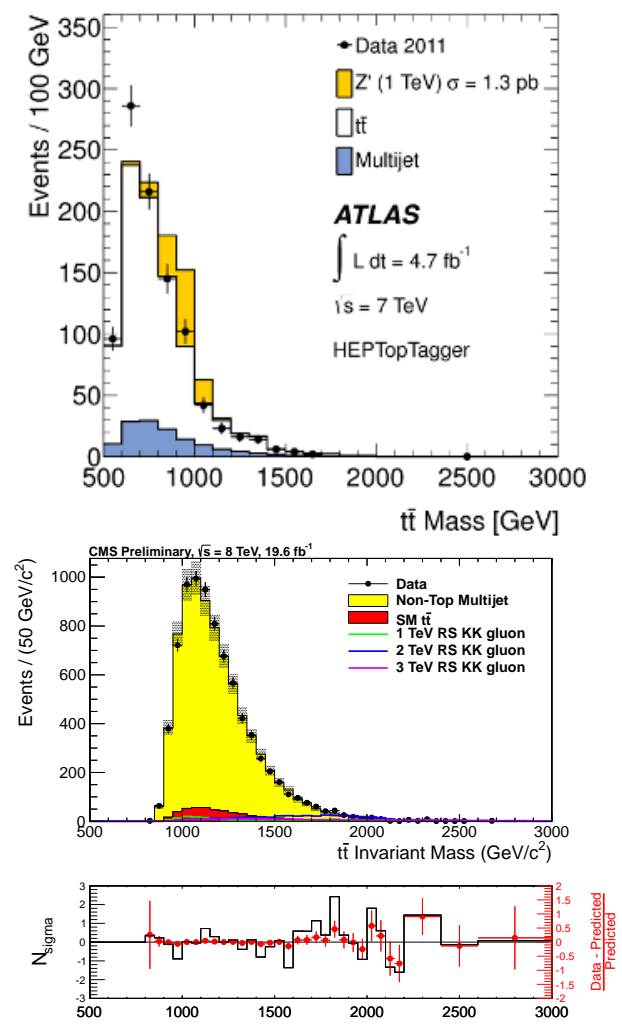

Figure 5. Comparison of $m_{t \bar{t}}$ distributions for the all-hadronic top pair resonance searches performed by ATLAS (top, figure from [14]) and CMS (bottom, figure from [9]).

As of this writing, the latest results from CMS include $19.6 \mathrm{fb}^{-1}$ of $\sqrt{s}=8 \mathrm{TeV}$ collision data. Several signal models are considered; $1 \%(10 \%)$ width $Z^{\prime}$ bosons are excluded up to $1.65(2.35) \mathrm{TeV} / c^{2}$, while RS KK gluons are excluded up to $1.8 \mathrm{TeV} / c^{2}$. The latest results from ATLAS analyze $4.7 \mathrm{fb}^{-1}$ of $\sqrt{s}=7 \mathrm{TeV}$ data, excluding narrow $Z^{\prime}$ bosons with masses between 1.0 and $1.32 \mathrm{TeV} / c^{2}$, and $\mathrm{KK}$ gluons with masses below $1.62 \mathrm{TeV} / c^{2}$. In addition, the CMS search reports a limit on general enhancements to the $m_{t \bar{t}}$ distribution, excluding models which predict more than 1.79 times the expected Standard Model $t \bar{t}$ event rate (assuming the same cuts of the specific analysis).

\subsection{Top Partner Searches}

Apart from searches for resonances in the $m_{t \bar{t}}$ spectrum, there are several searches with unique final states involving top quarks. This section will detail some of those analyses.

CMS has recently performed an analysis searching for exotic partners of the top quark, having electric charge $5 e / 3$, known as $T_{5 / 3}$. Physics models including the $T_{5 / 3}$, such as KK gluon models, can solve the hierarchy problem and are also compatible with the observed Higgs boson mass. With the expected decay $T_{5 / 3} \rightarrow t W$, the final state of this specific search [15] consists of a pair of leptons having the same electric charge, and can also contain boosted $W$ bosons or boosted top quarks in the final state. The event selection consists of requiring 2 same- 


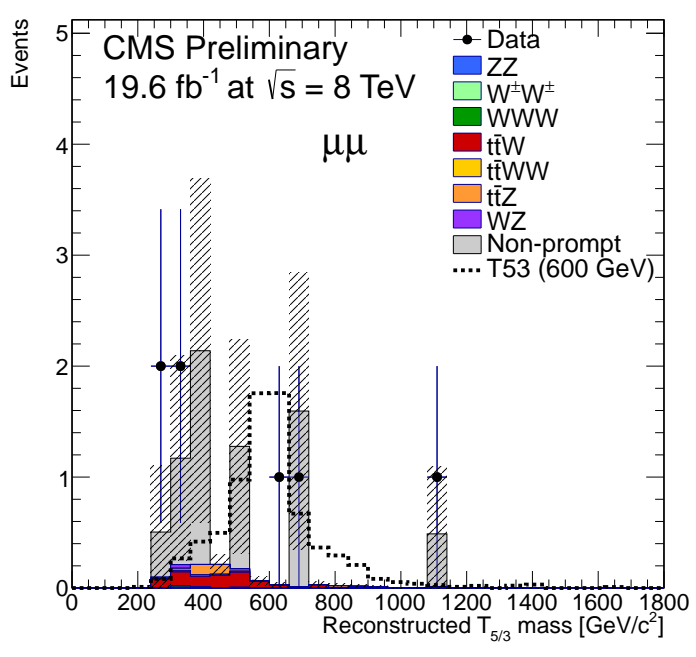

Figure 6. Reconstructed mass distribution of $T_{5 / 3}$ candidates, in the same-sign dimuon channel. Figure from Ref. [15].

charge leptons with $p_{T}>30 \mathrm{GeV} / c$, and 5 or more "jet constituents". This counting aims to count the number of partons in the final state - for example, a boosted top quark contributes 3 to this count when being reconstructed in a single jet. A boosted $W$ boson counts as 2 , and a light quark jet counts as just one. In addition to a requirement on this count, quarkonia and $Z$ vetos are imposed, and an $H_{T}>900 \mathrm{GeV}$ requirement is imposed. After the full event selection, the $T_{5 / 3}$ mass is reconstructed and its distribution is used to exclude this particle with masses up to $770 \mathrm{GeV} / c^{2}$, using $19.6 \mathrm{fb}^{-1}$ of data collected at $\sqrt{s}=8$ TeV. Figure 6 shows the reconstructed $T_{5 / 3}$ mass distribution for the dimuon selection.

ATLAS performs a similar search in the same-sign dilepton final state [16], but studies several different signal hypotheses and models. These include fourth generation quarks, vector-like quarks, four-top contact interactions, and models producing same-sign top quark pair production. After an event selection requiring two same-sign leptons, two or more jets (at least 1 being consistent with the decay of a $b$-quark), missing transverse energy greater than $40 \mathrm{GeV}, H_{T}>550 \mathrm{GeV}$, and the imposition of quarkonia and $Z$ boson vetos, ATLAS excludes vector-like $B$ quarks with masses up to $590 \mathrm{GeV} / c^{2}$, and vector-like $T$ quarks with masses up to $540 \mathrm{GeV} / c^{2}$. In addition, $\mathrm{KK}$ gluons are excluded up to $900 \mathrm{GeV} / c^{2}$. These results are obtained using $14.3 \mathrm{fb}^{-1}$ of data collected at $\sqrt{s}=8 \mathrm{TeV}$.

In addition to the same-sign dilepton final state, ATLAS recently released results searching for vector-like quarks in the lepton+jets final state [17]. Vector-like top quark partners have many possible decay modes, such as $t^{\prime} \rightarrow b W, t Z, t H$, which can give distinct final states rich in $b$-jets and boosted vector bosons. Events containing one electron or muon and 6 or more jets are selected, and further divided according to the number of identified $b$ quark jets. The main background to this search is Standard Model $t \bar{t}$ production. After the full event selection,

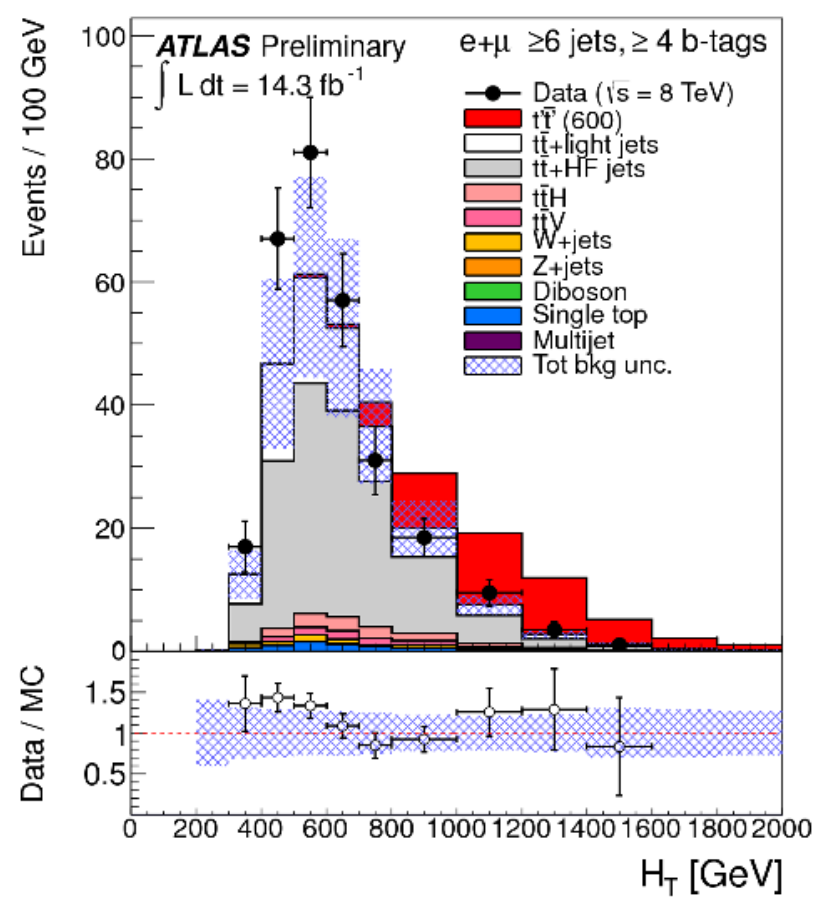

Figure 7. $H_{T}$ distribution for events in the vector-like $t^{\prime}$ quark search containing four or more jets identified as being consistent with a $b$-quark decay. The signal, shown by the solid red histogram, is isolated very well in this sub-channel of the analysis. Figure from Ref. [17].

the $H_{T}$ distribution is used for signal discrimination. Figure 7 shows the $H_{T}$ distribution for events containing 4 or more identified $b$-quark jets.

With this analysis including $14.3 \mathrm{fb}^{-1}$ of $2012 \mathrm{LHC}$ data, ATLAS excludes vector-like $t^{\prime}$ quarks with masses up to $790 \mathrm{GeV} / c^{2}$ in the context of an SU(2) doublet model, and $640 \mathrm{GeV} / c^{2}$ for an SU(2) singlet model. Furthermore, a scan is done over all the possible branching ratios $t^{\prime} \rightarrow b W, t Z$, and $t H$, so that any specific model can be analyzed and exclusion limits obtained for various scenarios.

Finally, there is recent progress by CMS in the search for an excited top quark partner, $t^{\star}$ which decays to a top quark and associated gluon [18]. The event selection for this analysis consists of a high-quality electron or muon, 6 or more jets (at least one consistent with a $b$-quark decay), and missing transverse energy due to the leptonic decay of the top quark. Kinematic constraints are used to reconstruct the $t^{\star}$ mass using the various objects reconstructed in the detector. A fit is performed using the data distribution to estimate the total background contribution after the event selection. The reconstructed mass distribution $\left(m_{t+g}\right)$ is then used as the signal discriminant for limit setting. After the full event selection and the combination of muon and electron channels, this analysis excludes excited top quarks $t^{\star}$ with masses up to $794 \mathrm{GeV} / c^{2}$, using $19.6 \mathrm{fb}^{-1}$ of data collected at $\sqrt{s}=8 \mathrm{TeV}$. Figure 8 shows the results of this analysis. 

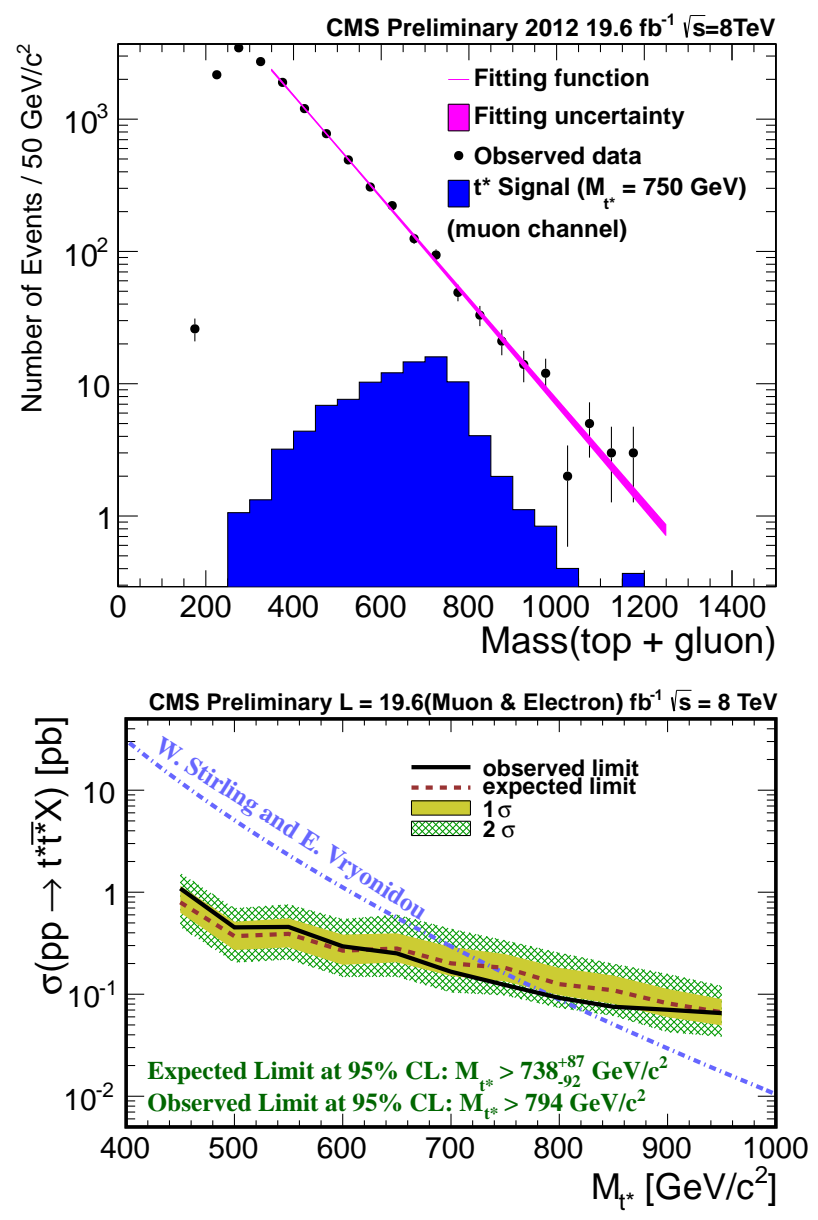

Figure 8. Top: Reconstructed $m_{t+g}$ distribution for the muon event selection. A fit is done to the data to determine the background contribution. The expected signal can be seen in the blue histogram. Bottom: Upper limits obtained in the search for $t^{\star} \rightarrow t g$ pair production. For the combination of muon and electron channels, the $t^{\star}$ mass can be excluded up to $794 \mathrm{GeV} / c^{2}$. Figures from Ref. [18].

\section{Conclusion}

This report has summarized many analyses searching for new physics involving top quark reconstruction in the final state, including $t \bar{t}$ resonances, and exotic top quark partners like $T_{5 / 3}$ and $t^{\star}$. With the large sample of top quarks produced by the LHC, the reconstruction techniques used are able to be validated. This validation provides the understanding necessary for extending these reconstruction techniques to searches for new physics producing top quarks in the final state.

Many of the LHC analyses are pushing mass exclusion limits above the $1 \mathrm{TeV} / c^{2}$ mark. In this regime, one cannot simply rely on standard reconstruction techniques, but must begin to take advantage of specialized algorithms, such as the top tagging algorithms described in this report. These algorithms have enhanced the sensitivity of several of the searches detailed here, and will become critically important to maintain sensitivity as the searches utilize the upcoming dataset to be collected at a higher centerof-mass energy.

Going forward, we must continue to explore the top sector, as many new physics models predict particles with large couplings to the top quark. The next run of the LHC will be an exciting time for many of these analyses. Much work will be required, but a discovery may be waiting!

For more information on the individual analyses presented here, please see the contributions to the conference proceedings from parallel talks, as well as the individual documentation for each analysis, listed in the references of this report. Thanks very much to the organizers of LHCP for a great conference!

\section{References}

[1] K. Agashe, A. Belyaev, T. Krupovnickas, G. Perez, J. Virzi, Phys. Rev. D 77, 015003 (2008)

[2] R.M. Harris, C.T. Hill, S.J. Parke (1999), hep-ph/9911288

[3] G. Aad et al. (ATLAS Collaboration), Journal of Instrumentation 3, S08003 (2008)

[4] S. Chatrchyan et al. (CMS Collaboration), Journal of Instrumentation 3, S08004 (2008)

[5] CMS Collaboration, CMS-PAS-JME-10-013 (2011)

[6] T. Plehn, M. Spannowsky, M. Takeuchi, Phys.Rev. D85, 034029 (2012), 1111.5034

[7] ATLAS Collaboration, ATLAS-CONF-2012-065 (2012)

[8] D.E. Kaplan, K. Rehermann, M.D. Schwartz, B. Tweedie, Phys. Rev. Lett. 101, 142001 (2008), 0806.0848

[9] CMS Collaboration, CMS-PAS-B2G-12-005 (2013)

[10] CMS Collaboration, CMS-PAS-B2G-12-006 (2013)

[11] ATLAS Collaboration, ATLAS-CONF-2013-052 (2013)

[12] ATLAS Collaboration, ATLAS-CONF-2011-123 (2011)

[13] CMS Collaboration, CMS-PAS-TOP-11-010 (2011)

[14] G. Aad et al. (ATLAS Collaboration), JHEP 1301, 116 (2013), 1211.2202

[15] CMS Collaboration, CMS-PAS-B2G-12-012 (2013)

[16] ATLAS Collaboration, ATLAS-CONF-2013-051 (2013)

[17] ATLAS Collaboration, ATLAS-CONF-2013-018 (2013)

[18] CMS Collaboration, CMS-PAS-B2G-12-014 (2012) 